\title{
Expression profile of circulating microRNAs as a promising fingerprint for cervical cancer diagnosis and monitoring
}

\author{
WENHUI JIA $^{1 *}$, YUANZHE WU ${ }^{2 *}$, QIN ZHANG ${ }^{2}$, GE GAO $^{1}$, CHENYU ZHANG $^{1}$ and YANG XIANG ${ }^{1}$ \\ ${ }^{1}$ State Key Laboratory of Pharmaceutical Biotechnology, School of Life Sciences, Nanjing University, Nanjing, \\ Jiangsu 210093; ${ }^{2}$ Department of Gynaecology and Obstetrics, Jinling Hospital, Clinical School of Medical College, \\ Nanjing University, Nanjing, Jiangsu 210002, P.R. China
}

Received May 28, 2014; Accepted April 22, 2015

DOI: $10.3892 / \mathrm{mco} .2015 .560$

\begin{abstract}
Sensitive and specific biomarkers for the early detection of cervical cancer are urgently required to reduce the high morbidity and mortality of this disease. We previously demonstrated that circulating microRNAs (miRNAs) are correlated with certain types of human cancer. The aim of this study was to investigate the altered profile of serum miRNAs in cervical cancer patients in order to predict cervical cancer at a relative early stage. Serum samples were collected from 213 cervical cancer patients and 158 age- and ethnicity-matched controls. An initial screening of miRNA expression was performed by Solexa sequencing. Differential expression was validated using the stem-loop miRNA quantitative polymerase chain reaction (qPCR) assay in individual samples and the samples were arranged by two-phase selection and validation. The Solexa sequencing results revealed 12 markedly upregulated serum miRNAs in cervical cancer patients compared with controls. The reverse transcription-qPCR analysis identified a profile of 5 serum miRNAs (miR-21, -29a, -25, -200a and -486-5p) as a cervical cancer biomarker. The receiver operating characteristic curves indicated that a panel of 5 miRNAs constitutes a more sensitive and specific diagnostic test compared with any single miRNA-based assay, the squamous cell carcinoma antigen or the carbohydrate antigen 125 . More importantly, miR-29a and miR-200a may indicate tumor histological grade
\end{abstract}

Correspondence to: Professor Yang Xiang, State Key Laboratory of Pharmaceutical Biotechnology, School of Life Sciences, Nanjing University, 22 Hankou Road, Nanjing, Jiangsu 210093, P.R. China E-mail: xiangy@nju.edu.cn

*Contributed equally

Abbreviations: miRNA, microRNA; Pap, Papanicolaou test; RT-qPCR, reverse transcription-quantitative polymerase chain reaction; CIN, cervical intraepithelial neoplasia; SCC, squamous cell carcinoma; ROC, receiver operating characteristic; AUC, area under the curve; RSF, risk score function; $95 \%$ CI, 95\% confidence interval; HPV, human papillomavirus

Key words: cervical cancer, diagnosis, microRNAs, non-invasive biomarker, serum and progression stage. Therefore, a 5-miRNA signature identified from genome-wide serum miRNA expression profiling may serve as a fingerprint for cervical cancer diagnosis.

\section{Introduction}

Cervical cancer is the third most commonly diagnosed cancer and the fourth leading cause of cancer-related mortality in women worldwide, accounting for $9 \%(529,800)$ of total new cancer cases and $8 \%(275,100)$ of total cancer deaths among women in 2008 (1). Cervical cancer is also the major cause of death in women of reproductive age (2-4). Cervical cancer is characterized by a long period of preclinical disease progressing through a number of well-defined premalignant stages. The transition of normal epithelium to preneoplastic cervical intraepithelial neoplasia (CIN) and invasive cervical cancer may require $>10$ years (5). The overall survival was reported to be $\sim 80-90 \%$ for stage $\mathrm{Ib}, 70-80 \%$ for stage II, $60 \%$ for stage III and $15 \%$ for stage IVa disease (6). From a clinical perspective, if detected prior to the point of progression to invasive disease, a variety of treatment options are available and the disease is almost certainly curable. Unfortunately, the early stages of cervical cancer are usually asymptomatic. When common signs and symptoms (such as vaginal bleeding and/or discharge and pelvic or back pain) appear, the disease is usually at an advanced stage. Furthermore, a number of these symptoms are non-specific and may represent a variety of different conditions (7).

Cervical carcinoma in situ is confined to the epithelial layer; therefore, the Papanicolaou test (Pap smear) cannot effectively detect the lesion. A technology assessment of cervical cytology concluded that conventional Pap smear screening had a specificity of $98 \%$, but sensitivity of only $51 \%$ (8). Pap smear screening is not highly effective at detecting adenocarcinoma, or its precursors. Colposcopy and random biopsies may diagnose some early-stage cervical cancer patients, although the invasiveness of these diagnostic procedures limits their efficacy. CIN is asymptomatic and essentially unrecognizable on gross inspection or palpation (9). Squamous cell carcinoma (SCC) antigen and carbohydrate antigen 125 (CA125) are two serum tumor biomarkers commonly used in clinical practice to detect and monitor cervical cancer; however, neither of these two makers is specific to this malignancy. In addition, 


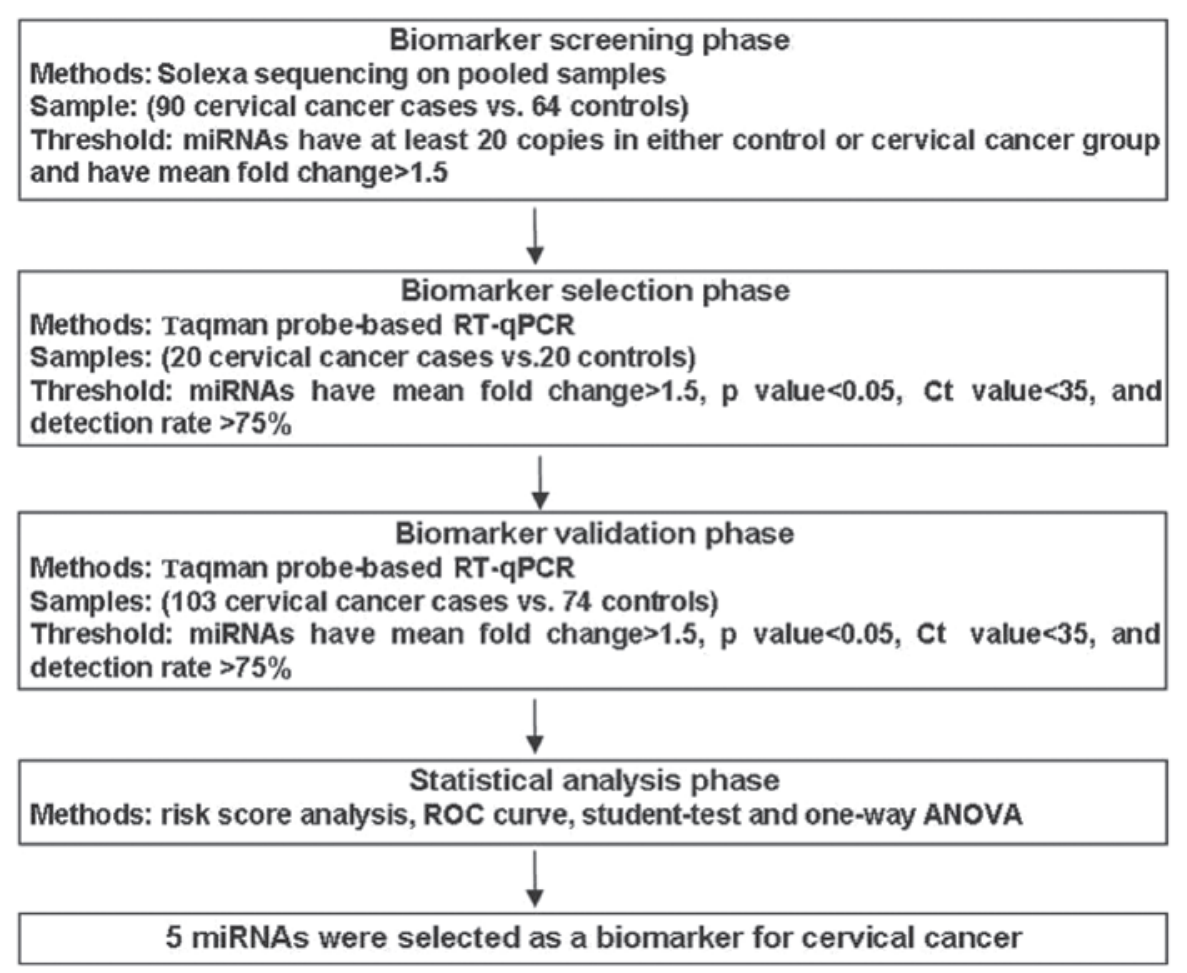

Figure 1. Flow chart of the experimental design. miRNAs, microRNAs; RT-qPCR, reverse transcription-quantitative polymerase chain reaction; ROC, receiver operating characteristic; ANOVA, analysis of variance.

both markers have a poor sensitivity for early-stage cervical cancer (26 and $23 \%$, respectively) $(10,11)$.

Specific and sensitive non-invasive biomarkers for the detection of human epithelial malignancy are urgently required to reduce the worldwide morbidity and mortality of cervical cancer. MicroRNAs (miRNAs) are 19-24-nt non-coding RNAs that are frequently deregulated in cancer and have shown great promise as tissue-based markers for cancer classification (12-14). Recent studies have also demonstrated that the differential expression of tissue miRNAs in cervical cancer may be of substantial diagnostic and prognostic value $(15,16)$.

The surgical collection of tissue samples is an invasive procedure. By contrast, serum sample collection is an easy and cost-effective method. We systematically demonstrated that the unique expression patterns of these circulating miRNAs are correlated with certain human diseases (17-23). However, the global miRNA pattern in the sera of cervical cancer patients has not yet been determined.

By analyzing the genome-wide miRNA expression profile using Solexa sequencing and the stem-loop miRNA quantitative polymerase chain reaction (qPCR) assay, we aimed to determine a unique panel of miRNAs that are differentially expressed in the serum of cervical cancer patients.

\section{Materials and methods}

Study design, patients and controls. All the samples were collected from consenting individuals according to the protocols approved by the Ethics Committee of the First Affiliated Hospital of Nanjing Medical University (Nanjing, China). A multistage case-control study was designed to identify a serum miRNA profile for cervical cancer (Fig. 1). In total, 213 patients with primary cervical cancer and 158 control subjects were enrolled in our study. In the initial screening stage, cervical cancer serum samples pooled from 90 cervical cancer patients and control donors pooled from 64 normal samples were subjected to Solexa sequencing to identify the miRNAs. We subsequently performed a confirmation analysis with a hydrolysis probe-based reverse transcription-qPCR (RT-qPCR) assay to refine the number of serum miRNAs in the cervical cancer signature (123 cervical cancer and 94 control samples). This analysis was performed in two phases: i) Training set, serum samples from 20 cervical cancer patients and 20 controls; and ii) validation set, an additional 103 cervical cancer serum samples and 74 normal subjects. All the patients were diagnosed with cervical carcinoma and treated at the Jinling Hospital between March, 2010 and December, 2011. Blood samples were collected prior to any therapeutic procedures, such as surgery, chemotherapy or radiotherapy. The results were histopathologically confirmed following surgical resection of the tumors and tumor staging was performed according to criteria of the International Federation of Gynecology and Obstetrics. For patients who were unsuitable for surgical management, the histopathological characteristics and tumor stage were confirmed by histobiopsy and imaging technology. Control participants were recruited from a large pool of individuals undergoing a routine health checkup at the Jinling Hospital (Nanjing, China). The demographics and clinical characteristics of the patients in the training and validation sets are listed in Table I. The controls were matched to the patients by age and ethnicity. None of the healthy controls had previously been diagnosed with malignancy.

Sample processing and RNA extraction. Serum separation was performed by centrifugation of the blood samples at $3,000 \mathrm{xg}$ 
Table I. Demographic and clinical characteristics of the patients and control individuals in the training and validation sets.

\begin{tabular}{|c|c|c|c|}
\hline Variables & $\begin{array}{c}\text { Cases, } \\
\text { no. }(\%) \\
(\mathrm{n}=123)\end{array}$ & $\begin{array}{c}\text { Controls, } \\
\text { no. }(\%) \\
(n=94)\end{array}$ & P-value \\
\hline $\begin{array}{l}\text { Age, years } \\
(\text { mean } \pm \text { SD })\end{array}$ & $46.0 \pm 8.6$ & $47.8 \pm 7.5$ & 0.350 \\
\hline$\geq 55$ & $20(16.2)$ & $18(19.1)$ & 0.303 \\
\hline $45-54$ & $61(50.0)$ & $53(56.4)$ & \\
\hline$<45$ & $42(33.8)$ & $23(24.5)$ & \\
\hline Marital status & & & 0.892 \\
\hline Married & $123(100)$ & $93(98.9)$ & \\
\hline Unmarried & $0(0.0)$ & $1(1.1)$ & \\
\hline Menopausal status & & & 0.248 \\
\hline Postmenopausal & $27(21.9)$ & $18(19.1)$ & \\
\hline Premenopausal & $77(62.6)$ & $53(56.4)$ & \\
\hline Unknown & $19(15.5)$ & $23(24.5)$ & \\
\hline FIGO stage & & & - \\
\hline $0(\mathrm{CIN})$ & $38(31.4)$ & & \\
\hline I & $59(47.0)$ & & \\
\hline II & $24(19.6)$ & & \\
\hline III & $2(2)$ & & \\
\hline IV & $0(0)$ & & \\
\hline $\begin{array}{l}\text { Histological } \\
\text { differentiation }\end{array}$ & & & - \\
\hline Moderate or high & $39(31.7)$ & & \\
\hline Poor & $25(20.6)$ & & \\
\hline $\mathrm{CIN}$ & $38(30.9)$ & & \\
\hline Undetermined & $21(16.8)$ & & \\
\hline Tumor histology & & & - \\
\hline Adenocarcinoma & $8(6.5)$ & & \\
\hline $\mathrm{SCC}$ & $114(92.7)$ & & \\
\hline Clear cell carcinoma & $1(0.8)$ & & \\
\hline $\begin{array}{l}\text { Significant cardiac } \\
\text { dysfunction }\end{array}$ & & & 0.813 \\
\hline Yes & $3(2.4)$ & $1(1.1)$ & \\
\hline No & 120 (97.6) & $93(98.9)$ & \\
\hline Hypertension & & & 0.106 \\
\hline Yes & $10(8.1)$ & $1(1.1)$ & \\
\hline No & $113(91.9)$ & $93(98.9)$ & \\
\hline $\begin{array}{l}\text { Neurological disease } \\
\text { or diabetes }\end{array}$ & & & 0.599 \\
\hline Yes & $1(0.8)$ & $0(0.0)$ & \\
\hline No & $122(99.2)$ & $94(100)$ & \\
\hline
\end{tabular}

aStudent's t-test. ${ }^{\mathrm{b}}$ Two-sided $\chi^{2}$ test. FIGO, International Federation of Gynecology and Obstetrics; CIN, cervical intraepithelial neoplasia; SCC, squamous cell carcinoma.

for $10 \mathrm{~min}$, followed by a 15 -min high-speed centrifugation at $12,000 \times$ g. Subsequently, the supernatant sera were stored at $-80^{\circ} \mathrm{C}$ until further analysis.
Table II. Information of cervical cancer patients and healthy controls in Solexa sequencing.

\begin{tabular}{lcc}
\hline Variables & $\begin{array}{c}\text { Cervical cancer } \\
\text { cases, no. }(\%) \\
(\mathrm{n}=90)\end{array}$ & $\begin{array}{c}\text { Controls, } \\
\text { no. }(\%) \\
(\mathrm{n}=64)\end{array}$ \\
\hline $\begin{array}{l}\text { Age, years } \\
(\text { mean } \pm \text { SD })\end{array}$ & $46.0 \pm 9.3$ & $46.0 \pm 7.1$ \\
$\geq 55$ & $24(26.5)$ & $13(20.3)$ \\
$45-54$ & $42(47.0)$ & $27(42.2)$ \\
$<45$ & $24(26.5)$ & $24(37.5)$ \\
Histological & & \\
differentiation & & \\
Poor & $9(10.5)$ & \\
Moderate & $50(55.3)$ & \\
High & $31(34.2)$ & \\
FIGO stage & & \\
0 (CIN) & $33(36.4)$ & \\
I & $45(50.0)$ & \\
II & $11(12.1)$ & \\
III & $1(1.5)$ & \\
\hline
\end{tabular}

FIGO, International Federation of Gynecology and Obstetrics; CIN, cervical intraepithelial neoplasia.

For the Solexa sequencing assay, equal volumes of sera from 90 patients with cervical cancer $(0.67 \mathrm{ml}$ each $)$ and 64 controls with similar age and gender distributions $(0.94 \mathrm{ml}$ each) were pooled separately to form the case and control sample pools (Table II). TRIzol reagent (Invitrogen Life Technologies, Carlsbad, CA, USA) was used according to the manufacturer's instructions with minor modifications to extract total RNA from each pool of serum samples $(\sim 60 \mathrm{ml})$. The aqueous phase was subjected to three steps of acid phenol/chloroform purification to eliminate protein residues prior to isopropyl alcohol precipitation. The resulting RNA pellet was dissolved in $20 \mu \mathrm{l}$ diethylpyrocarbonate-treated water and stored at $-80^{\circ} \mathrm{C}$ until further analysis.

For the RT-qPCR assay, total RNA was extracted from $100 \mu \mathrm{l}$ serum with a one-step phenol/chloroform purification protocol. In brief, $100 \mu \mathrm{l}$ serum was mixed with $200 \mu \mathrm{l}$ acid phenol, $200 \mu \mathrm{l}$ chloroform and $300 \mu \mathrm{l}$ diethylpyrocarbonate-treated water. The mixture was vortex-mixed vigorously and incubated at room temperature for $15 \mathrm{~min}$. Following phase separation, the aqueous layer was mixed with $40 \mu \mathrm{l}$ sodium acetate ( $3 \mathrm{~mol} / \mathrm{l}, \mathrm{pH} 5.3$ ) and $800 \mu 1$ isopropyl alcohol. This solution was stored at $-20^{\circ} \mathrm{C}$ for $1 \mathrm{~h}$. The RNA pellet was collected by centrifugation at $16,000 \mathrm{x}$ g for $20 \mathrm{~min}$ at $4{ }^{\circ} \mathrm{C}$. The resulting RNA pellet was washed once with $750 \mathrm{ml} / 1$ ethanol and dried for $10 \mathrm{~min}$ at room temperature. Finally, the pellet was dissolved in $20 \mu \mathrm{l}$ of ribonuclease-free water and stored at $-80^{\circ} \mathrm{C}$ until further analysis.

Solexa sequencing and in silico analysis. First, total RNA was extracted as mentioned above. Using PAGE purification, all the small RNA molecules $(<30 \mathrm{bp})$ were isolated. After 
ligating a pair of adaptors to their $5^{\prime}$ and $3^{\prime}$ ends, the small RNA molecules were amplified for 17 cycles and then $~ 90$-bp fragments were isolated from agarose gels. The Illumina genome analyzer (Illumina, San Diego, CA, USA) was used for cluster generation and sequencing analysis according to the manufacturer's instructions. Finally, the reads were processed for in silico analysis as previously described (23).

$R T-q P C R$. Briefly, total RNA ( $2 \mu \mathrm{l})$ was reverse-transcribed to cDNA using AMV reverse transcriptase (Takara Biomedical Technology, Dalian, China) and the stem-loop RT primer (Applied Biosystems, Foster City, CA, USA). qPCR was performed using TaqMan miRNA probes (Applied Biosystems) on the Applied Biosystems 7300 Sequence Detection system. All the reactions were run in triplicate and the $\mathrm{Ct}$ values were determined using the fixed threshold settings. U6 and 5S rRNA are degraded in serum samples and there is no current consensus on housekeeping miRNAs for RT-qPCR analysis of serum miRNAs; furthermore, our group observed that the expression level of the mixture of let- $7 \mathrm{i},-7 \mathrm{~g}$ and $-7 \mathrm{~d}$ is rather stable in human serum (24). Therefore, the miRNA expression level was normalized to the mixture of let-7i, $-7 \mathrm{j}$ and $-7 \mathrm{~d}$ in our study. The relative expression levels of target miRNAs were determined by the $2^{-\Delta \Delta C t}$ equation, in which $\Delta \mathrm{Ct}$ was calculated as follows: $\Delta \mathrm{Ct}=\mathrm{Ct}_{\text {miR-of-interest }}-\mathrm{Ct}_{\text {let-7. }}$. The miRNA expression levels were also normalized to the serum volume in this study.

SCC antigen and CA125 determination. The serum levels of SCC antigen and CA125 were measured by chemiluminescence immunoassay using an ARCHITECT ${ }^{\mathrm{TM}}$ i2000SR Access Immunoassay system (Abott, Lake Forest, IL, USA).

Statistical analysis. Quantitative data are presented as means \pm standard deviation. Statistical significance was determined using the Student's t-test. $\mathrm{P}<0.05$ was considered to indicate a statistically significant difference. For each miRNA, we constructed a receiver operating characteristic (ROC) curve and calculated the area under the ROC curve (AUC) to evaluate the specificity and sensitivity of cervical cancer prediction. We performed a risk score analysis to evaluate the associations between cervical cancer and serum miRNA expression level. The risk score of each miRNA, denoted as $\mathrm{s}$, was set to 1 if the expression level was higher than the upper $95 \%$ confidence interval (95\% CI) for the corresponding miRNA level in controls and to 0 otherwise. A risk score function (RSF) to predict cervical cancer risk was defined according to a linear combination of the expression level for each miRNA. For example, the RSF for sample i using information from 5 miRNAs was calculated as follows:

$$
\mathrm{RSF}_{\mathrm{i}}=\sum_{\mathrm{j}-1}^{5} \mathrm{~W}_{\mathrm{j}} \cdot \mathrm{s}_{\mathrm{ij}}
$$

In this equation, $\mathrm{s}_{\mathrm{ij}}$ is the risk score for miRNA $\mathrm{j}$ on sample $\mathrm{i}$ and $\mathrm{W}_{\mathrm{j}}$ is the weight of the risk score of miRNA $\mathrm{j}$. To determine $\mathrm{W}_{\mathrm{s}}$, five univariate logistic regression models were fitted using the disease status with each of the risk scores. The regression coefficient of each risk score was used as the weight to indicate the contribution of each miRNA to the RSF. Frequency tables and ROC curves were then used to evaluate the diagnostic effects of the profiling and to determine the appropriate
Table III. Differentially expressed microRNAs (miRNAs) in cervical cancer patient serum samples compared to controls, as determined by Solexa sequencing.

\begin{tabular}{lrrc}
\hline miRNAs & $\begin{array}{c}\text { Copy no. in } \\
\text { cervical } \\
\text { cancer }\end{array}$ & $\begin{array}{c}\text { Copy } \\
\text { no. in } \\
\text { controls }\end{array}$ & $\begin{array}{c}\text { Cervical } \\
\text { cancer/ } \\
\text { healthy }\end{array}$ \\
\hline hsa-miR-21 & 677 & 101 & 6.70 \\
hsa-miR-29a & 1,159 & 253 & 4.58 \\
hsa-miR-486-5p & 23,699 & 9,509 & 2.49 \\
hsa-miR-25 & 114 & 46 & 2.49 \\
hsa-miR-146b-5p & 346 & 159 & 2.18 \\
hsa-miR-423-3p & 52 & 26 & 2.00 \\
hsa-miR-140-3p & 455 & 235 & 1.94 \\
hsa-miR-101 & 61 & 32 & 1.90 \\
hsa-miR-26b & 36 & 21 & 1.72 \\
hsa-miR-191 & 179 & 109 & 1.64 \\
hsa-miR-29c & 61 & 37 & 1.64 \\
hsa-miR-200a & 32 & 21 & 1.50 \\
\hline
\end{tabular}

cut-off point. All the statistical analyses were performed with Statistical Analysis system software, version 9.1.3 (SAS Institute, Cary, NC, USA).

\section{Results}

Clinical characteristics of the patients. All the patients enrolled in the present study had been clinically and pathologically diagnosed with cervical cancer. There was no significant difference in the distribution of age, marital and menopausal status between the cancer patients and the controls. In general, cervical cancer patients and controls had no other diseases, including significant cardiac dysfunction, hypertension, neurological disorders or diabetes at the time of blood sample collection (Table I).

Solexa sequencing of serum miRNAs in cervical cancer. The Solexa data revealed that miRNAs were the major components of small RNAs ( $<30 \mathrm{bp})$ in the serum. The expression of a miRNA was considered 'significantly altered' only if $\geq 20$ copies were detected by Solexa sequencing, together with a $>1.5$-fold change in its expression level between the patient and control groups. Based on these criteria, the 12 miRNAs found to be differentially expressed in cervical cancer were further analyzed by RT-qPCR (Table III).

Confirmation of miRNA production by RT-qPCR analysis. We used the RT-qPCR assay to confirm the expression of candidate miRNAs. In the training set, miRNAs were measured in a separate set of individual serum samples from 20 cervical cancer patients and 20 healthy controls; only miRNAs with a mean change $>1.5$-fold and a $\mathrm{P}<0.05$ were selected for further analysis (Fig. 1). This phase generated a list of 5 miRNAs that had a significant differential expression pattern, namely miR-21, -29a, -200a, -25 and -486-5p. Compared to their levels in control samples, these 5 miRNAs were increased by 
Table IV. Differentially expressed serum microRNAs (miRNAs) in cervical cancer cases compared to controls in the training and validation sets.

\begin{tabular}{|c|c|c|c|c|c|c|}
\hline \multirow[b]{2}{*}{ miRNAs } & \multicolumn{3}{|c|}{ Training set (fold-change \pm SD) } & \multicolumn{3}{|c|}{ Validation set (fold-change $\pm \mathrm{SD}$ ) } \\
\hline & $\begin{array}{c}\text { Controls } \\
(\mathrm{n}=20)\end{array}$ & $\begin{array}{l}\text { Cervical cancer } \\
\quad(\mathrm{n}=20)\end{array}$ & P-value & $\begin{array}{c}\text { Controls } \\
(n=74)\end{array}$ & $\begin{array}{l}\text { Cervical cancer } \\
\quad(n=103)\end{array}$ & P-value \\
\hline miR-21 & $1 \pm 0.06$ & $3.99 \pm 0.82$ & $4.8 \times 10^{-3}$ & $1 \pm 0.06$ & $4.42 \pm 1.00$ & $3.8 \times 10^{-3}$ \\
\hline miR-29a & $1 \pm 0.07$ & $1.70 \pm 0.35$ & $3.5 \times 10^{-2}$ & $1 \pm 0.05$ & $2.04 \pm 0.17$ & $3.9 \times 10^{-6}$ \\
\hline miR-200a & $1 \pm 0.06$ & $1.88 \pm 0.44$ & $3.3 \times 10^{-2}$ & $1 \pm 0.05$ & $1.65 \pm 0.24$ & $9.3 \times 10^{-3}$ \\
\hline $\operatorname{miR}-25$ & $1 \pm 0.16$ & $2.12 \pm 0.44$ & $1.1 \times 10^{-2}$ & $1 \pm 0.07$ & $3.63 \pm 0.72$ & $3.6 \times 10^{-3}$ \\
\hline miR-486-5p & $1 \pm 0.07$ & $2.62 \pm 0.72$ & $1.8 \times 10^{-2}$ & $1 \pm 0.09$ & $3.18 \pm 0.72$ & $3.0 \times 10^{-3}$ \\
\hline
\end{tabular}

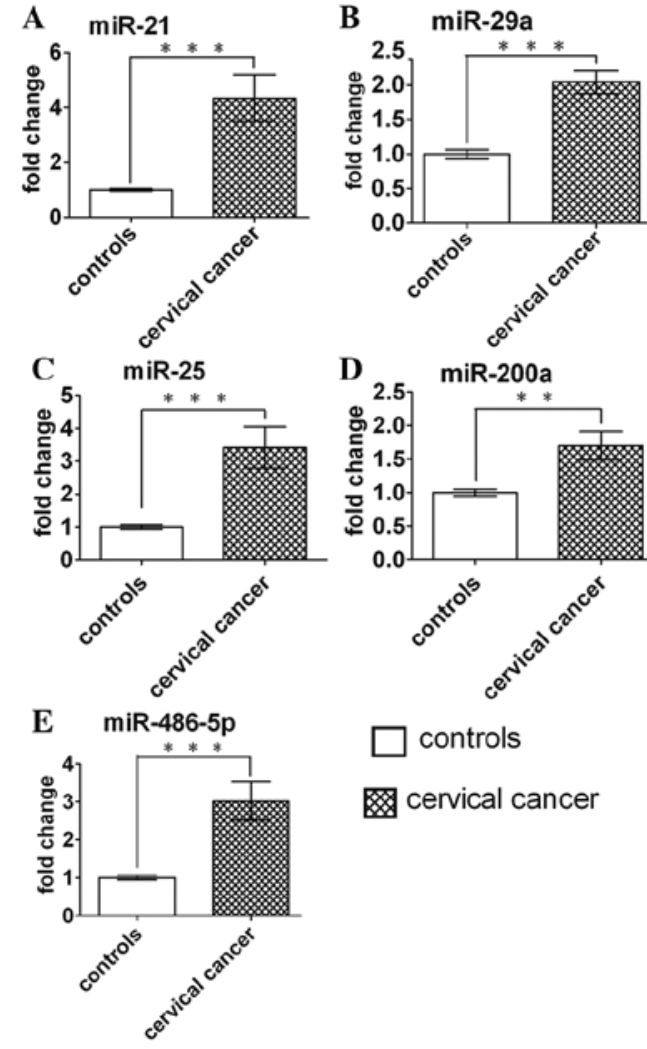

Figure 2. miRNA expression in cervical cancer patients and control subjects. (A-E) The serum levels of the 5 miRNAs were measured in 123 cervical cancer cases and 94 healthy controls (in the training and the validation sets) using a hydrolysis probe-based reverse transcription-quantitative polymerase chain reaction assay. ${ }^{* * *} \mathrm{P}<0.01$ and ${ }^{* * * *} \mathrm{P}<0.001$.

3.99-, 1.70-, 1.88-, 2.12- and 2.62-fold, respectively, in cervical cancer samples (Table IV).

These 5 miRNAs were further examined by RT-qPCR in a larger cohort including 103 cervical cancer patients and 74 matched controls. The miRNA expression pattern alterations in the validation set were consistent with those in the training set (Table IV). The differences in concentration for the 5 miRNAs in 123 cervical cancer patients and 94 control subjects enrolled in the training and validation sets are shown in Fig. 2.

Risk score and ROC curve analysis. To further evaluate the diagnostic value of the 5-miRNA profiling system, we used
Table V. Risk score analysis of cervical cancer and control subjects on the 5-microRNA profile.

\begin{tabular}{lcccc}
\hline Group & $0-0.50$ & $0.50-2.00$ & PPV & NPV \\
\hline Control & 83 & 11 & - & 0.78 \\
Cervical cancer & 23 & 100 & 0.90 & - \\
\hline
\end{tabular}

PPV, positive predictive value; NPV, negative predictive value.

a risk score formula to calculate RSF for cervical cancer and control samples. The samples were ranked according to their RSF and then divided into a high-risk group (predicted cervical cancer cases) and a low-risk group (control individuals). The frequency table and the ROC curves were then used to evaluate the diagnostic effect of the 5-miRNA profiling system and determine the appropriate cut-off point.

The ROC curves constructed to compare the relative concentrations of the 5 miRNAs in the cervical cancer patients and healthy controls yielded the following AUCs: miR-21, 0.819 (95\% CI: 0.762-0.876); miR-29a, 0.819 (95\% CI: 0.762-0.876); miR-25, 0.726 (95\% CI: 0.656-0.795); miR-200a, 0.658 (95\% CI: 0.575-0.728) and miR-486-5p, 0.685 (95\% CI: 0.610-0.759) (Fig. 3A-E). Among the 5 miRNAs investigated, miR-21 displayed the highest sensitivity and specificity for cervical cancer diagnosis. To illustrate the contribution of individual serum miRNAs to the AUC of the ROC curve, we established ROC curves to evaluate the diagnostic value of each miRNA for differentiating between cervical cancer cases and controls. We found that the subsequent addition of each of the 5 miRNAs incrementally improved the sensitivity and specificity of the miRNA-based biomarkers in discriminating cervical cancer cases from controls (Fig. 3F). The AUC value of the combination of 5 miRNAs [0.908 (95\% CI: 0.868-0.948)], was markedly higher compared with that of the SCC antigen [0.655 (95\% CI: 0.541-0.770)] and CA125 [0.570 (95\% CI: 0.418-0.722)] (Fig.3G and H). With an optimal cut-off value, in which the sum of the sensitivity and specificity was maximal, the specificity was 88.6 and the sensitivity was $81.0 \%$. The positive and negative predictive values were 0.90 and 0.78 , respectively (Table V). The results 

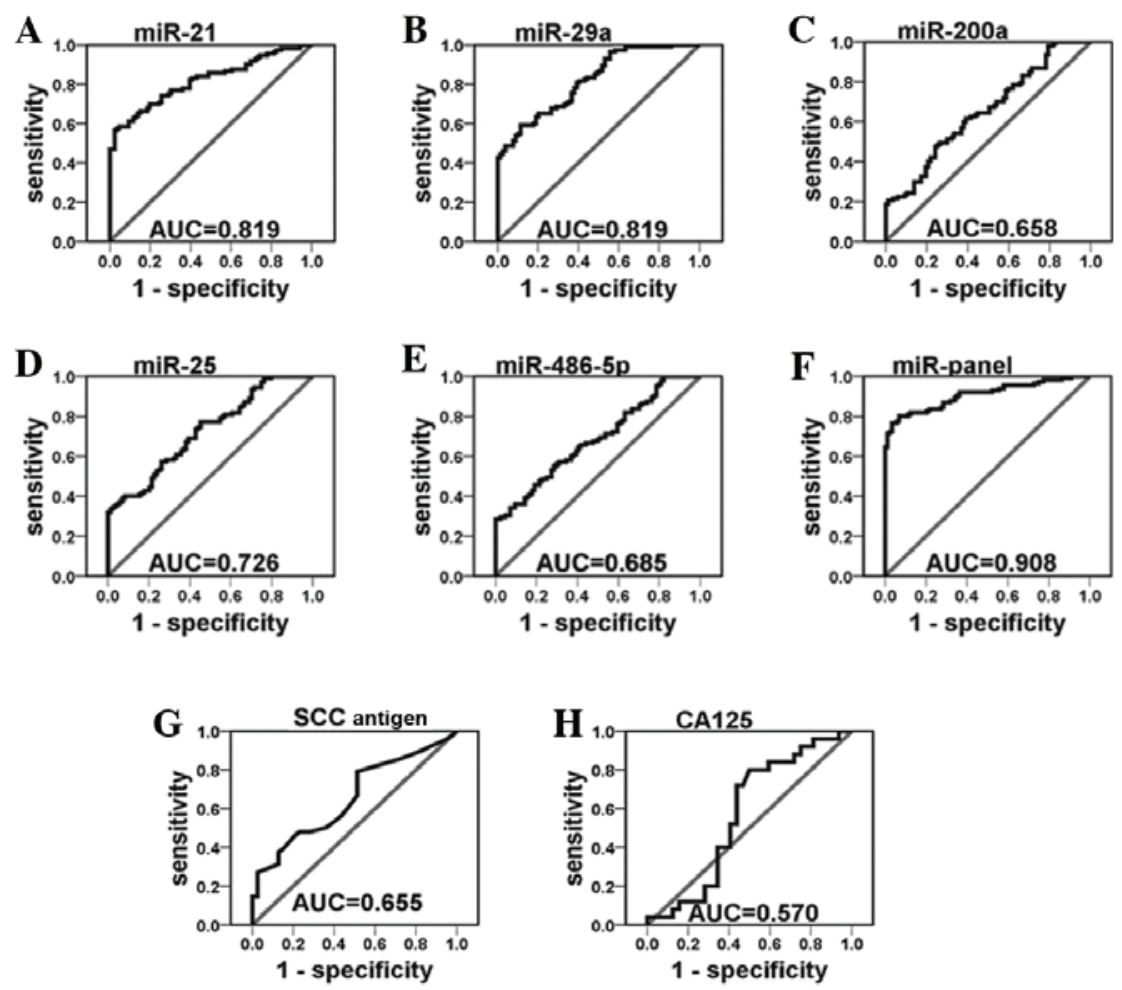

Figure 3. Receiver operating characteristic curves for the ability of the serum concentations of (A-E) the 5 individual miRNAs; (F) the 5-miRNA panel; (G) squamous cell carcinoma (SCC) antigen; and (H) carbohydrate antigen 125 (CA125) to differentiate between cervical cancer cases ( $\mathrm{n}=123)$ and controls $(\mathrm{n}=94)$. AUC, area under the curve.
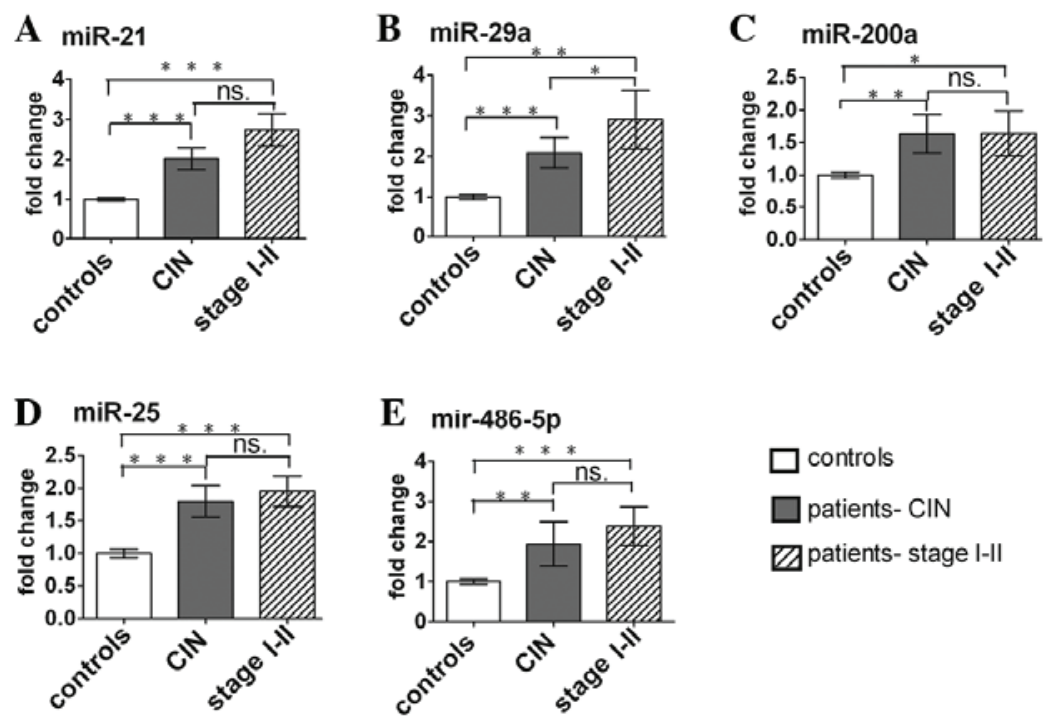

Figure 4. Expression levels of the selected serum miRNAs in cervical cancer at different stages. (A-E) Fold-change of the 5 miRNAs in the serum of cervical cancer patients from CIN to stages I and II. ${ }^{*} \mathrm{P}<0.05,{ }^{* * *} \mathrm{P}<0.01$ and ${ }^{* * * *} \mathrm{P}<0.001$. CIN, cervical intraepithelial neoplasia; ns, not significant.

indicated that the 5-serum miRNA signature is more reliable compared with any single miRNA-based assay, the SCC antigen or CA125 in the diagnosis of cervical cancer.

Altered serum miRNAs by different stage and differentiation type. The expression levels of the 5 serum miRNAs in cervical cancer patients at different stages and different differentiation types were analyzed. The serum miRNAs in the cervical cancer patients increased significantly in CIN cases compared with normal controls. The fold-change of miR-21, -29a, -200a, -25 and -486-5p was 2.02, 2.10, 1.64, 1.84 and 1.94, respectively (Fig. 4). As shown in Fig. 4B, the serum miR-29a level was progressively higher from earlier-stage disease (CIN) to later stages (I and II). In addition, miR-29a and miR-200a also exhibited a distinct upregulation in poorly differentiated cases compared with patients with well- or moderately differentiated tumors. 


\section{Discussion}

miRNAs in human serum and other body fluids remain stable after being subjected to harsh conditions, under which most RNAs would be degraded (25-27). Possible explanations for the remarkable stability of miRNAs in the serum are that they are protected by binding proteins or microvesicles and that they may be chemically modified (e.g., methylation) $(26,27)$. Several studies have suggested that active secretion by cells is a major source of serum miRNAs. Furthermore, recent studies revealed the novel genetic exchange between cells using miRNAs, either in microvesicles $(\leq 1 \mu \mathrm{m})$ or in small membrane vesicles of endocytic origin, referred to as exosomes (50-100 nm) (28-30). The secreted miRNAs contained in exosomes may also be transferred from tumor cells to tumor cells, or from tumor cells to normal cells, indicating that an oncogene may be propagated horizontally through exosomes (31). In addition to this high stability, the characteristics of miRNAs, such as tissue-specific miRNA signatures and the availability of several copies per cell, indicate potential advantages as biomarkers compared with other nucleic acids, such as circulating DNA and mRNA $(32,33)$. Thus, previous findings support that circulating miRNAs may be used as non-invasive diagnostic markers.

Despite differences in pathogenesis, tumors share common characteristics, such as unlimited proliferation and rapid metastasis. The upregulation of certain miRNAs is likely to be observed in the sera of patients with tumors. In this study, we systematically demonstrated that serum miR-21, -29a, $-25,-200 \mathrm{a}$ and $-486-5 \mathrm{p}$ may serve as a non-invasive, accurate biomarkers for cervical cancer diagnosis. The ROC curves indicated that a panel of 5 miRNAs has great potential as a more sensitive and specific diagnostic test compared with any single miRNA-based assay, the SCC antigen and CA125 for cervical cancer.

The functional study of miRNAs in tumor tissue may also be helpful for evaluating serum miRNAs as indicators of various types of cancer. Hu et al (16) suggested that miR-200a is potentially involved in tumor control by regulating cancer cell metastasis. Zhang et al (34) demonstrated that miR-25 directly regulates apoptosis by targeting Bim in ovarian cancer. miR-486-5p may function as a novel tumor suppressor miRNA in gastric cancer and its anti-oncogenic activity may involve the direct targeting and inhibition of olfactomedin 4 (35). Such findings suggested that serum miRNAs may play a pivotal and general role as signaling molecules in physiological and pathological events.

Patients with CIN may undergo extensive hysterectomy, which is likely curative in these patients. We further focused on whether these miRNAs may be used as diagnostic markers for early cervical cancer. These 5 miRNAs were clearly upregulated in cervical cancer patient serum samples compared with control samples and exhibited an average $\sim 1.8$-fold change in patients with CIN, whereas the Pap smear is relatively inefficient. More importantly, miR-29a and miR-200a may be of value for clinical monitoring and prognosis. The results suggested a potential application in diagnosing cervical cancer at an early stage, even at the precancerous lesion stage.

In 1995 the World Health Organization declared human papillomavirus (HPV) as a known carcinogen for cervical cancer, as the DNA of mucosal high-risk HPV types was detected in almost all cervical cancers (36). The elucidation of unidentified genetic alterations due to HPV and miRNA interactions may shed more light on the mechanistic underpinnings of HPV-induced oncogenesis (37). HPVs exhibit oncogenic properties, at least in part by reshaping the milieu of cellular miRNAs and miR-29a is associated with HPV E6/E7 expression in vivo at the pre-neoplastic stage (38). Hence, our results may also be helpful in investigating the mechanisms underlying the development of HPV-infected malignant neoplasms in the future.

In summary, our study identified a panel of 5 serum miRNAs as a signature for cervical cancer detection at its early stages. miR-29a and miR-200a were also clearly upregulated in poorly differentiated cases compared with patients with well- or moderately differentiated tumors. These results may provide impetus for the clinical value of serum miRNAs in predicting the prognosis of cervical cancer.

\section{Acknowledgements}

This study was supported by National Natural Science Foundation of China (grant nos. 30570731, 30871195, 81070653, 81270907, 81370926, J1103512 and J1210026).

\section{References}

1. Siegel R, Naishadham D, Jemal A, et al: Cancer statistics, 2012. CA Cancer J Clin 62: 10-29, 2012.

2. Parkin DM, Almonte M, Bruni L, et al: Burden and trends of type-specific human papillomavirus infections and related diseases in the Latin America and Caribbean region. Vaccine 26 (Suppl 11): L1-L15, 2008.

3. Mathew A and George PS: Trends in incidence and mortality rates of squamous cell carcinoma and adenocarcinoma of cervix - worldwide. Asian Pac J Cancer Prev 10: 645-650, 2009.

4. Vizcaino, AP, Moreno V, Bosch FX, et al: International trends in incidence of cervical cancer: II. Squamous-cell carcinoma. Int J Cancer 86: 429-435, 2000.

5. Richart RM: A modified terminology for cervical intraepithelial neoplasia. Obstet Gynecol 75: 131-133, 1990.

6. Mayr NA, Small W Jr and Gaffney DK: Cervical cancer. In Decision Making in Radiation Oncology. Lu JJ and Brady LW (eds).Vol 2. Springer-Verlag, Berlin Heidelberg, pp661-701, 2011.

7. Kyrgiou M and Mahmood SI: Invasive cancer of the cervix Obstet Gynecol Reprod Med 20: 147-154, 2010.

8. Wright TC Jr, Schiffman M, Solomon D, et al: Interim guidance for the use of human papillomavirus DNA testing as an adjunct to cervical cytology for screening. Obstet Gynecol 103: 304-309, 2004.

9. Baker JJ: Conventional and liquid-based cervicovaginal cytology: a comparison study with clinical and histologic follow-up. Diagn Cytopathol 27: 185-188, 2002.

10. Avall-Lundqvist EH, Sjövall K, Nilsson BR, et al: Prognostic significance of pretreatment serum levels of squamous cell carcinoma antigen and CA 125 in cervical carcinoma. Eur J Cancer 28A: 1695-1702, 1992.

11. Bolli JA, Doering DL, Bosscher JR, et al: Squamous cell carcinoma antigen: clinical utility in squamous cell carcinoma of the uterine cervix. Gynecol Oncol 55: 169-173, 1994.

12. Bartel DP: MicroRNAs: genomics, biogenesis, mechanism, and function. Cell 116: 281-297, 2004.

13. Calin GA and Croce CM: MicroRNA signatures in human cancers. Nat Rev Cancer 6: 857-866, 2006.

14. Lee JW, Choi CH, Choi JJ, et al: Altered microRNA expression in cervical carcinomas. Clin Cancer Res 14: 2535-2542, 2008.

15. Pereira PM, Marques JP, Soares AR, et al: MicroRNA expression variability in human cervical tissues. PLoS One 5: e11780, 2010.

16. Hu X, Schwarz JK, Lewis JS Jr, et al: A microRNA expression signature for cervical cancer prognosis. Cancer Res 70: 1441-1448, 2010. 
17. Yang C, Wang $\mathrm{C}$, Chen $\mathrm{X}$, et al: Identification of seven serum microRNAs from a genome-wide serum microRNA expression profile as potential noninvasive biomarkers for malignant astrocytomas. Int J Cancer 132: 116-127, 2013.

18. Li C, Fang Z, Jiang T, et al: Serum microRNAs profile from genome-wide serves as a fingerprint for diagnosis of acute myocardial infarction and angina pectoris. BMC Med Genomics 6: 16, 2013.

19. Li LM, Hu ZB, Zhou ZX, et al: Serum microRNA profiles serve as novel biomarkers for $\mathrm{HBV}$ infection and diagnosis of $\mathrm{HBV}$-positive hepatocarcinoma. Cancer Res 70: 9798-9807, 2010.

20. Chen $X, H u Z$, Wang W, et al: Identification of ten serum microRNAs from a genome-wide serum microRNA expression profile as novel noninvasive biomarkers for nonsmall cell lung cancer diagnosis. Int J Cancer 130: 1620-1628, 2012.

21. Liu R, Zhang C, Hu Z, et al: A five-microRNA signature identified from genome-wide serum microRNA expression profiling serves as a fingerprint for gastric cancer diagnosis. Eur J Cancer 47: 784-791, 2011

22. Zhang C, Wang C, Chen X, et al: Expression profile of microRNAs in serum: a fingerprint for esophageal squamous cell carcinoma. Clin Chem 56: 1871-1879, 2010.

23. Chen X, Li Q, Wang J, et al: Identification and characterization of novel amphioxus microRNAs by Solexa sequencing. Genome Biol 10: R78, 2009.

24. Chen X, Liang H, Guan D, et al: A Combination of Let-7d, Let-7g and Let-7i serves as a stable reference for normalization of serum microRNAs. PLoS ONE 8: e79652. (Epub ahead of print) doi:10.1371/journal.pone.0079652, 2013.

25. Chim SS, Shing TK, Hung EC, et al: Detection and characterization of placental microRNAs in maternal plasma. Clin Chem 54: 482-490, 2008.

26. Chen X, Ba Y, Ma L, et al: Characterization of microRNAs in serum: a novel class of biomarkers for diagnosis of cancer and other diseases. Cell Res 18: 997-1006, 2008.
27. Mitchell PS, Parkin RK, Kroh EM, et al: Circulating microRNAs as stable blood-based markers for cancer detection. Proc Natl Acad Sci USA 105: 10513-10518, 2008.

28. Cocucci E, Racchetti G and Meldolesi J: Shedding microvesicles: artefacts no more. Trends Cell Biol 19: 43-51, 2009.

29. Ghosh AK, Secreto CR, Knox TR, et al: Circulating microvesicles in B-cell chronic lymphocytic leukemia can stimulate marrow stromal cells: implications for disease progression. Blood 115: 1755-1764, 2010.

30. Valadi H, Ekström K, Bossios A, et al: Exosome-mediated transfer of mRNAs and microRNAs is a novel mechanism of genetic exchange between cells. Nat Cell Biol 9: 654-659, 2007.

31. Kosaka N, Iguchi H and Ochiya T: Circulating microRNA in body fluid: a new potential biomarker for cancer diagnosis and prognosis. Cancer Sci 101: 2087-2092, 2010.

32. Calin GA and Croce CM: MicroRNA-cancer connection: the beginning of a new tale. Cancer Res 66: 7390-7394, 2006.

33. Lu J, Getz G, Miska EA, et al: MicroRNA expression profiles classify human cancers. Nature 435: 834-838, 2005.

34. Zhang H, Zuo Z, Lu X, et al: MiR-25 regulates apoptosis by targeting Bim in human ovarian cancer. Oncol Rep 27: 594-598, 2012.

35. Oh HK, Tan AL, Das K, et al: Genetic loss of miR-486 regulates tumor progression and the OLMF4 antiapoptotic factor in gastric cancer. Clin Cancer Res 17: 2657-2667, 2011.

36. Walboomers JM, Jacobs MV, Manos MM, et al: Human papillomavirus is a necessary cause of invasive cervical cancer worldwide. J Pathol 189: 12-19, 1999.

37. Martinez I, Gardiner AS, Board KF, et al: Human papillomavirus type 16 reduces the expression of microRNA-218 in cervical carcinoma cells. Oncogene 27: 2575-2582, 2008.

38. Li Y, Wang F, Xu J, et al: Progressive miRNA expression profiles in cervical carcinogenesis and identification of HPV-related target genes for miR-29. J Pathol 224: 484-495, 2011. 